\title{
A joint search for gravitational wave bursts with AURIGA and LIGO
}

(The AURIGA Collaboration)

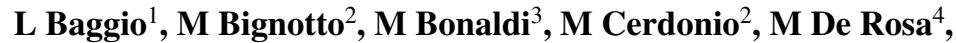

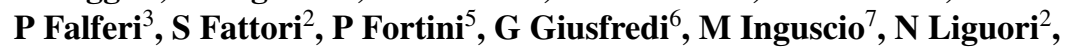
S Longo ${ }^{8}$, F Marin ${ }^{7}$, R Mezzena ${ }^{1}$, A Mion ${ }^{1}$, A Ortolan ${ }^{8}$, S Poggi ${ }^{9}$,

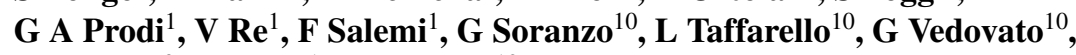
A Vinante ${ }^{3}, \mathbf{S}$ Vitale $^{1}, \mathbf{J}$ P Zendri ${ }^{10}$
\end{abstract}

(The LIGO Scientific Collaboration)

B Abbott ${ }^{11}$, R Abbott ${ }^{11}$, R Adhikari ${ }^{11}$, J Agresti ${ }^{11}$, P Ajith ${ }^{12}$, B Allen ${ }^{12,13}$, R Amin ${ }^{14}$, S B Anderson ${ }^{11}$, W G Anderson ${ }^{13}$, M Arain ${ }^{15}$, M Araya ${ }^{11}$, H Armandula ${ }^{11}$, M Ashley ${ }^{16}, \mathbf{S}$ Aston $^{17}$, P Aufmuth ${ }^{18}$, C Aulbert ${ }^{19}$, S Babak ${ }^{19}$, S Ballmer ${ }^{11}$, H Bantilan ${ }^{20}$, B C Barish ${ }^{11}$, C Barker ${ }^{21}$, D Barker ${ }^{21}$, B Barr ${ }^{22}$, P Barriga ${ }^{23}$, M A Barton ${ }^{22}$, K Bayer ${ }^{24}$, K Belczynski $^{25}$, J Betzwieser ${ }^{24}$, P T Beyersdorf ${ }^{26}$, B Bhawal ${ }^{11}$, I A Bilenko ${ }^{27}$, G Billingsley ${ }^{11}$, R Biswas ${ }^{13}$, E Black $^{11}$, K Blackburn ${ }^{11}$, L Blackburn $^{24}$, D Blair ${ }^{23}$, B Bland ${ }^{21}$, J Bogenstahl ${ }^{22}$, L Bogue ${ }^{28}$, R Bork $^{11}$, V Boschi ${ }^{11}$, S Bose ${ }^{29}$, P R Brady ${ }^{13}$, V B Braginsky ${ }^{27}$, J E Brau ${ }^{30}$, M Brinkmann ${ }^{12}$, A Brooks ${ }^{31}$, D A Brown ${ }^{11}$, A Bullington ${ }^{33}$, A Bunkowski ${ }^{12}$, A Buonanno ${ }^{34}$, O Burmeister ${ }^{12}$, D Busby ${ }^{11}$, W E Butler ${ }^{35}$, R L Byer ${ }^{33}$, L Cadonati ${ }^{24}$, G Cagnoli ${ }^{22}$, J B Camp ${ }^{36}$, J Cannizzo $^{36}$, K Cannon ${ }^{13}$, C A Cantley ${ }^{22}, \mathbf{J ~ C a o}^{24}$, L Cardenas ${ }^{11}$, K Carter ${ }^{28}$, M M Casey ${ }^{22}$, G Castaldi ${ }^{37}$, C Cepeda ${ }^{11}$, E Chalkley $^{22}$, P Charlton $^{38}$, S Chatterji $^{11}$, S Chelkowski ${ }^{12}$, Y Chen ${ }^{19}$, F Chiadini ${ }^{39}$, D Chin ${ }^{40}$, E Chin ${ }^{23}$, J Chow ${ }^{16}$, N Christensen ${ }^{20}$, J Clark ${ }^{22}$, P Cochrane ${ }^{12}$, T Cokelaer ${ }^{41}, \mathbf{C}$ N Colacino ${ }^{17}$, R Coldwell ${ }^{15}$, R Conte $^{39}$, D Cook $^{21}$, T Corbitt $^{24}$, D Coward ${ }^{23}$, D Coyne ${ }^{11}$, J D E Creighton ${ }^{13}$, T D Creighton ${ }^{11}$, R P Croce ${ }^{37}$, D R M Crooks ${ }^{22}$, A M Cruise ${ }^{17}$, A Cumming $^{22}$, J Dalrymple ${ }^{42}$, E D'Ambrosio ${ }^{11}$, K Danzmann ${ }^{12,18}$,

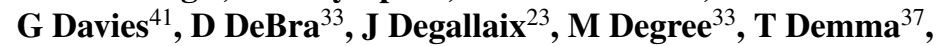
V Dergachev ${ }^{40}, \mathbf{S}$ Desai $^{43}$, R DeSalvo ${ }^{11}$, S Dhurandhar ${ }^{44}$, M Díaz $^{45}$, J Dickson ${ }^{16}$, A Di Credico ${ }^{42}$, G Diederichs ${ }^{18}$, A Dietz ${ }^{41}$, E E Doomes ${ }^{46}$, R W P Drever ${ }^{47}$, J-C Dumas ${ }^{23}$, R J Dupuis ${ }^{11}$, J G Dwyer ${ }^{48}$, P Ehrens ${ }^{11}$,

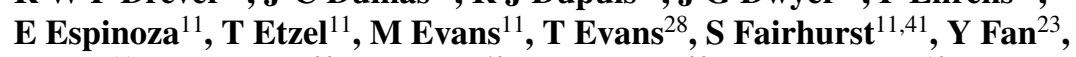
D Fazi ${ }^{11}$, M M Fejer ${ }^{33}$, L S Finn ${ }^{43}$, V Fiumara ${ }^{39}$, N Fotopoulos ${ }^{13}$, A Franzen $^{18}$, K Y Franzen ${ }^{15}$, A Freise ${ }^{17}$, R Frey ${ }^{30}$, T Fricke ${ }^{35}$,

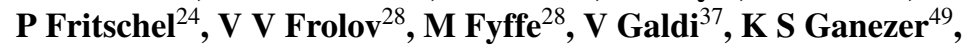
J Garofoli $^{21}$, I Gholami ${ }^{19}$, J A Giaime ${ }^{14,28}$, S Giampanis ${ }^{35}$, K D Giardina ${ }^{28}$, K Goda ${ }^{24}$, E Goetz ${ }^{40}$, L M Goggin ${ }^{11}$, G González ${ }^{14}$, S Gossler $^{16}$,

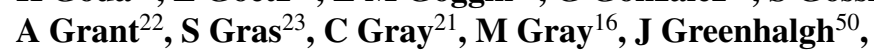

A M Gretarsson ${ }^{51}$, R Grosso ${ }^{45}$, H Grote ${ }^{12}$, S Grunewald ${ }^{19}$, M Guenther ${ }^{21}$, 
R Gustafson ${ }^{40}$, B Hage ${ }^{18}$, D Hammer ${ }^{13}$, C Hanna $^{14}$, J Hanson $^{28}$,

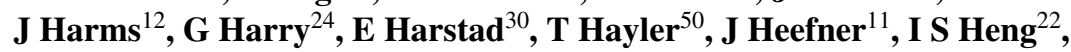
A Heptonstall ${ }^{22}$, M Heurs ${ }^{12}$, M Hewitson ${ }^{12}, \mathbf{S}$ Hild $^{18}$, E Hirose $^{42}$, D Hoak $^{28}$, D Hosken ${ }^{31}$, J Hough ${ }^{22}$, E Howell ${ }^{23}$, D Hoyland ${ }^{17}$,

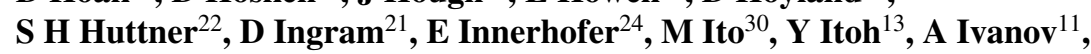
D Jackrel $^{33}$, B Johnson ${ }^{21}$, W W Johnson ${ }^{14}$, D I Jones ${ }^{52}$, G Jones ${ }^{41}$, R Jones ${ }^{22}$, L Ju ${ }^{23}$, P Kalmus ${ }^{48}$, V Kalogera ${ }^{25}$, D Kasprzyk ${ }^{17}$,

E Katsavounidis $^{24}$, K Kawabe ${ }^{21}$, S Kawamura ${ }^{53}$, F Kawazoe ${ }^{53}$, W Kells ${ }^{11}$, D G Keppel ${ }^{11}$, F Ya Khalili ${ }^{27}$, C Kim ${ }^{25}$, P King ${ }^{11}$, J S Kissel ${ }^{14}$,

S Klimenko ${ }^{15}$, K Kokeyama ${ }^{53}$, V Kondrashov ${ }^{11}$, R K Kopparapu ${ }^{14}$, D Kozak ${ }^{11}$, B Krishnan ${ }^{19}$, P Kwee ${ }^{18}$, P K Lam ${ }^{16}$, M Landry ${ }^{21}$, B Lantz ${ }^{33}$,

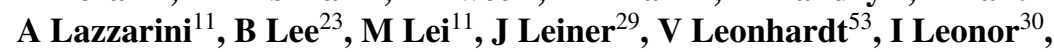
K Libbrecht $^{11}$, P Lindquist ${ }^{11}$, N A Lockerbie ${ }^{54}$, M Longo ${ }^{39}$, M Lormand $^{28}$, M Lubinski ${ }^{21}$, H Lück ${ }^{12}$, B Machenschalk ${ }^{19}$, M MacInnis ${ }^{24}$, M Mageswaran ${ }^{11}$, K Mailand ${ }^{11}$, M Malec $^{18}, \mathbf{V}$ Mandic $^{11}$, S Marano ${ }^{39}, \mathbf{S}$ Márka ${ }^{48}$, J Markowitz ${ }^{24}$, E Maros ${ }^{11}$, I Martin ${ }^{22}$, J N Marx ${ }^{11}, \mathbf{K}$ Mason $^{24}$, L Matone $^{48}, \mathbf{V ~ M a t t a ~}^{39}$, N Mavalvala ${ }^{24}$, R McCarthy ${ }^{21}$, D E McClelland ${ }^{16}$, S C McGuire ${ }^{46}$, M McHugh ${ }^{55}$, K McKenzie $^{16}$, J W C McNabb ${ }^{43}$, S McWilliams ${ }^{36}$, T Meier ${ }^{18}$, A Melissinos ${ }^{35}$, G Mendell ${ }^{21}$, R A Mercer ${ }^{15}$, S Meshkov ${ }^{11}$, C J Messenger ${ }^{22}$, D Meyers ${ }^{11}$, E Mikhailov ${ }^{24}$, S Mitra ${ }^{44}$, V P Mitrofanov ${ }^{27}$, G Mitselmakher ${ }^{15}$, R Mittleman $^{24}$, O Miyakawa ${ }^{11}$, S Mohanty ${ }^{45}$, G Moreno ${ }^{21}$, K Mossavi ${ }^{12}$, C MowLowry ${ }^{16}$, A Moylan ${ }^{16}$, D Mudge ${ }^{31}$, G Mueller ${ }^{15}$, S Mukherjee ${ }^{45}$, H Müller-Ebhardt ${ }^{12}$, J Munch ${ }^{31}$,

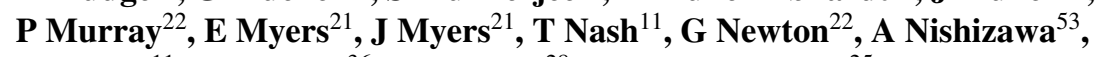
F Nocera ${ }^{11}$, K Numata ${ }^{36}$, B O'Reilly ${ }^{28}$, R O'Shaughnessy ${ }^{25}$, D J Ottaway ${ }^{24}$, H Overmier ${ }^{28}$, B J Owen ${ }^{43}$, Y Pan $^{34}$, M A Papa ${ }^{13}$, V Parameshwaraiah ${ }^{21}$, C Parameswariah $^{28}$, P Patel $^{11}$, M Pedraza ${ }^{11}$, S Penn ${ }^{56}$, V Pierro ${ }^{37}$, I M Pinto ${ }^{37}$, M Pitkin ${ }^{22}$, H Pletsch $^{12}$, M V Plissi ${ }^{22}$, F Postiglione ${ }^{39}$, R Prix ${ }^{19}$, V Quetschke ${ }^{15}$, F Raab ${ }^{21}$, D Rabeling ${ }^{16}$, H Radkins ${ }^{21}$, R Rahkola ${ }^{30}$, N Rainer ${ }^{12}$, M Rakhmanov ${ }^{43}$, M Ramsunder ${ }^{43}$, K Rawlins ${ }^{24}$, S Ray-Majumder ${ }^{13}$, T Regimbau ${ }^{41}$, H Rehbein ${ }^{12}$, S Reid ${ }^{22}$, D H Reitze ${ }^{15}$, L Ribichini ${ }^{12}$, R Riesen ${ }^{28}$, K Riles ${ }^{40}$, B Rivera ${ }^{21}$, N A Robertson ${ }^{11,22}$, C Robinson ${ }^{41}$, E L Robinson ${ }^{17}$, S Roddy ${ }^{28}$, A Rodriguez ${ }^{14}$, A M Rogan ${ }^{29}$, J Rollins ${ }^{48}$, J D Romano ${ }^{41}$, J Romie ${ }^{28}$,

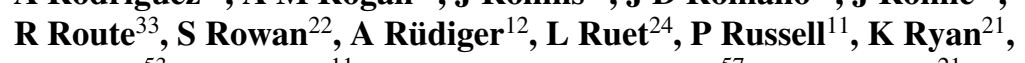
S Sakata $^{53}$, M Samidi ${ }^{11}$, L Sancho de la Jordana ${ }^{57}$, V Sandberg ${ }^{21}$, G H Sanders ${ }^{11}$, V Sannibale ${ }^{11}$, S Saraf ${ }^{58}$, P Sarin ${ }^{24}$, B S Sathyaprakash ${ }^{41}$, $\mathbf{S ~ S a t o}^{53}$, P R Saulson ${ }^{42}$, R Savage ${ }^{21}$, P Savov $^{32}$, A Sazonov $^{15}, \mathbf{S}$ Schediwy $^{23}$, R Schilling $^{12}$, R Schnabel ${ }^{12}$, R Schofield ${ }^{30}$, B F Schutz ${ }^{19,41}$, P Schwinberg ${ }^{21}$, S M Scott ${ }^{16}$, A C Searle ${ }^{16}$, B Sears ${ }^{11}$, F Seifert ${ }^{12}$, D Sellers ${ }^{28}$, A S Sengupta ${ }^{41}$, P Shawhan ${ }^{34}$, D H Shoemaker ${ }^{24}$, A Sibley ${ }^{28}$, X Siemens ${ }^{11,32}$, D Sigg ${ }^{21}$, S Sinha ${ }^{33}$, A M Sintes ${ }^{19,57}$, B J J Slagmolen ${ }^{16}$, J Slutsky ${ }^{14}$, J R Smith ${ }^{12}$, M R Smith ${ }^{11}$, K Somiya ${ }^{12,19}$, K A Strain ${ }^{22}$, D M Strom ${ }^{30}$, A Stuver ${ }^{43}$, T Z Summerscales ${ }^{59}$, K-X Sun ${ }^{33}$, M Sung ${ }^{14}$, P J Sutton ${ }^{11}$, H Takahashi ${ }^{19}$, D B Tanner ${ }^{15}$, M Tarallo ${ }^{11}$, R Taylor ${ }^{11}$, R Taylor ${ }^{22}$, J Thacker ${ }^{28}$, K A Thorne ${ }^{43}$, K S Thorne ${ }^{32}$, A Thüring ${ }^{18}$, M Tinto ${ }^{11}$, K V Tokmakov ${ }^{22}$, C Torres $^{45}$, C Torrie $^{22}$, G Traylor ${ }^{28}$, M Trias ${ }^{57}$, W Tyler ${ }^{11}$, D Ugolini ${ }^{60}$, C Ungarelli ${ }^{17}$, K Urbanek ${ }^{33}$, 
H Vahlbruch $^{18}$, M Vallisneri ${ }^{32}$, C Van Den Broeck ${ }^{41}$, M van Putten ${ }^{24}$, M Varvella ${ }^{11}$, S Vass ${ }^{11}$, A Vecchio ${ }^{17}$, J Veitch ${ }^{22}, \mathbf{P}$ Veitch $^{31}$, A Villar ${ }^{11}$, C Vorvick $^{21}$, S P Vyachanin ${ }^{27}$, S J Waldman ${ }^{11}$, L Wallace ${ }^{11}$, H Ward ${ }^{22}$, R Ward ${ }^{11}$, K Watts ${ }^{28}$, D Webber ${ }^{11}$, A Weidner ${ }^{12}$, M Weinert ${ }^{12}$, A Weinstein ${ }^{11}$, R Weiss ${ }^{24}, \mathbf{S}$ Wen $^{14}$, K Wette ${ }^{16}$, J T Whelan ${ }^{19}$, D M Whitbeck ${ }^{43}$, S E Whitcomb ${ }^{11}$, B F Whiting ${ }^{15}$, S Wiley ${ }^{49}$, C Wilkinson $^{21}$, P A Willems ${ }^{11}$, L Williams ${ }^{15}$, B Willke ${ }^{12,18}$, I Wilmut ${ }^{50}$, W Winkler ${ }^{12}$, C C Wipf ${ }^{24}$, S Wise ${ }^{15}$, A G Wiseman ${ }^{13}$, G Woan ${ }^{22}$, D Woods ${ }^{13}$, R Wooley ${ }^{28}$, J Worden $^{21}, \mathbf{W} \mathbf{W u}^{15}$, I Yakushin ${ }^{28}$,

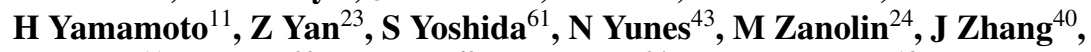
L Zhang $^{11}$, C Zhao ${ }^{23}$, N Zotov ${ }^{62}$, M Zucker ${ }^{24}, \mathbf{H}$ zur Mühlen ${ }^{18}$ and J Zweizig ${ }^{11}$

${ }^{1}$ Physics Department, University of Trento and INFN Gruppo Collegato di Trento, Padova Section, I-38050 Povo (Trento), Italy

2 INFN Padova Section and Department of Physics, University of Padova, I-35131 Padova, Italy

${ }^{3}$ Istituto di Fotonica e Nanotecnologie CNR-Fondazione Bruno Kessler and INFN Gruppo Collegato di Trento, Padova Section, I-38050 Povo (Trento), Italy

${ }^{4}$ INOA I-80078 Pozzuoli (Napoli), Italy and INFN Firenze Section, I-50121 Firenze, Italy

${ }^{5}$ Physics Department, University of Ferrara and INFN Ferrara Section, I-44100 Ferrara, Italy

${ }^{6}$ INOA, CNR, I-50125 Arcetri, Firenze, Italy

${ }^{7}$ LENS and Physics Department, University of Firenze and INFN Firenze Section,

I-50121 Firenze, Italy

${ }^{8}$ INFN, Laboratori Nazionali di Legnaro, I-35020 Legnaro (Padova), Italy

${ }^{9}$ Consorzio Criospazio Ricerche, I-38050 Povo (Trento), Italy

${ }^{10}$ INFN Padova Section, I-35100 Padova, Italy

${ }^{11}$ LIGO - California Institute of Technology, Pasadena, CA 91125, USA

12 Albert-Einstein-Institut, Max-Planck-Institut für Gravitationsphysik, D-30167 Hannover, Germany

${ }^{13}$ University of Wisconsin-Milwaukee, Milwaukee, WI 53201, USA

${ }^{14}$ Louisiana State University, Baton Rouge, LA 70803, USA

${ }^{15}$ University of Florida, Gainesville, FL 32611, USA

${ }^{16}$ Australian National University, Canberra, 0200, Australia

${ }^{17}$ University of Birmingham, Birmingham, B15 2TT, UK

${ }^{18}$ Leibnitz Universität Hannover, D-30167 Hannover, Germany

${ }^{19}$ Albert-Einstein-Institut, Max-Planck-Institut für Gravitationsphysik, D-14476 Golm, Germany

${ }^{20}$ Carleton College, Northfield, MN 55057, USA

${ }^{21}$ LIGO Hanford Observatory, Richland, WA 99352, USA

22 University of Glasgow, Glasgow, G12 8QQ, UK

${ }^{23}$ University of Western Australia, Crawley, WA 6009, Australia

${ }^{24}$ LIGO-Massachusetts Institute of Technology, Cambridge, MA 02139, USA

25 Northwestern University, Evanston, IL 60208, USA

${ }^{26}$ San Jose State University, San Jose, CA 95192, USA

${ }^{27}$ Moscow State University, Moscow, 119992, Russia

${ }^{28}$ LIGO Livingston Observatory, Livingston, LA 70754, USA

${ }^{29}$ Washington State University, Pullman, WA 99164, USA

${ }^{30}$ University of Oregon, Eugene, OR 97403, USA

${ }^{31}$ University of Adelaide, Adelaide, SA 5005, Australia

${ }^{32}$ Caltech-CaRT, Pasadena, CA 91125 , USA

${ }^{33}$ Stanford University, Stanford, CA 94305, USA

${ }^{34}$ University of Maryland, College Park, MD 20742 USA

35 University of Rochester, Rochester, NY 14627, USA

${ }^{36}$ NASA/Goddard Space Flight Center, Greenbelt, MD 20771, USA

${ }^{37}$ University of Sannio at Benevento, I-82100 Benevento, Italy

${ }^{38}$ Charles Sturt University, Wagga Wagga, NSW 2678, Australia

${ }^{39}$ University of Salerno, 84084 Fisciano (Salerno), Italy

${ }^{40}$ University of Michigan, Ann Arbor, MI 48109, USA

${ }^{41}$ Cardiff University, Cardiff, CF24 3AA, UK 
42 Syracuse University, Syracuse, NY 13244, USA

43 The Pennsylvania State University, University Park, PA 16802, USA

${ }^{44}$ Inter-University Centre for Astronomy and Astrophysics, Pune - 411007 , India

45 The University of Texas at Brownsville and Texas Southmost College, Brownsville, TX 78520, USA

${ }^{46}$ Southern University and A\&M College, Baton Rouge, LA 70813, USA

${ }^{47}$ California Institute of Technology, Pasadena, CA 91125, USA

${ }^{48}$ Columbia University, New York, NY 10027, USA

${ }^{49}$ California State University Dominguez Hills, Carson, CA 90747, USA

${ }^{50}$ Rutherford Appleton Laboratory, Chilton, Didcot, Oxon OX11 0QX, UK

${ }^{51}$ Embry-Riddle Aeronautical University, Prescott, AZ 86301, USA

52 University of Southampton, Southampton, SO17 1BJ, UK

${ }^{53}$ National Astronomical Observatory of Japan, Tokyo 181-8588, Japan

${ }^{54}$ University of Strathclyde, Glasgow, G1 1XQ, UK

${ }^{55}$ Loyola University, New Orleans, LA 70118, USA

${ }^{56}$ Hobart and William Smith Colleges, Geneva, NY 14456, USA

57 Universitat de les Illes Balears, E-07122 Palma de Mallorca, Spain

58 Rochester Institute of Technology, Rochester, NY 14623, USA

59 Andrews University, Berrien Springs, MI 49104 USA

60 Trinity University, San Antonio, TX 78212, USA

${ }^{61}$ Southeastern Louisiana University, Hammond, LA 70402, USA

${ }^{62}$ Louisiana Tech University, Ruston, LA 71272, USA

Received 18 October 2007, in final form 25 January 2008

Published 15 April 2008

Online at stacks.iop.org/CQG/25/095004

\section{Abstract}

The first simultaneous operation of the AURIGA detector ${ }^{63}$ and the LIGO observatory $^{64}$ was an opportunity to explore real data, joint analysis methods between two very different types of gravitational wave detectors: resonant bars and interferometers. This paper describes a coincident gravitational wave burst search, where data from the LIGO interferometers are cross-correlated at the time of AURIGA candidate events to identify coincident transients. The analysis pipeline is tuned with two thresholds, on the signal-to-noise ratio of AURIGA candidate events and on the significance of the cross-correlation test in LIGO. The false alarm rate is estimated by introducing time shifts between data sets and the network detection efficiency is measured by adding simulated gravitational wave signals to the detector output. The simulated waveforms have a significant fraction of power in the narrower AURIGA band. In the absence of a detection, we discuss how to set an upper limit on the rate of gravitational waves and to interpret it according to different source models. Due to the short amount of analyzed data and to the high rate of non-Gaussian transients in the detectors' noise at the time, the relevance of this study is methodological: this was the first joint search for gravitational wave bursts among detectors with such different spectral sensitivity and the first opportunity for the resonant and interferometric communities to unify languages and techniques in the pursuit of their common goal.

PACS numbers: $04.80 . \mathrm{Nn}, 95.30 . \mathrm{Sf}, 95.85 . \mathrm{Sz}$

\footnotetext{
${ }^{63}$ http://www.auriga.lnl.infn.it

${ }^{64} \mathrm{http}: / / \mathrm{www}$.ligo.org
} 


\section{Introduction}

Gravitational wave bursts are short duration perturbations of the spacetime metric due to such catastrophic astrophysical events as supernova core collapses [1] or the merger and ringdown phases of binary black hole coalescences [2,3]. Over the past decade, the search for these signals has been independently performed by individual detectors or by homogeneous networks of resonant bars [4] or laser interferometers [5-9]. The first coincident burst analysis between interferometers with different broadband sensitivity and orientation was performed by the TAMA and LIGO Scientific Collaborations [10]. That analysis required coincident detection of power excesses in at least two LIGO interferometers and in the TAMA detector in the 700-2000 Hz frequency band, where all sensitivities were comparable. The upper limit result accounted for the different antenna patterns with a Monte Carlo estimate of detection efficiency for sources uniformly distributed in the sky.

This paper describes a joint burst search in a heterogeneous network, comprised of LIGO and AURIGA. Although this is the first joint search for gravitational wave transients with interferometers and a resonant antenna, bar data were previously cross-correlated with LIGO data in the search for a gravitational wave stochastic background, with the ALLEGRO detector. That search provided what is, to date, the most competitive stochastic upper limit in the 905-925 Hz frequency band [14].

LIGO consists of three interferometers, two co-located in Hanford, WA, with $2 \mathrm{~km}$ and $4 \mathrm{~km}$ baselines and one in Livingston, LA, with a $4 \mathrm{~km}$ baseline, sensitive between $60 \mathrm{~Hz}$ and $4000 \mathrm{~Hz}$ with best performance in a $100 \mathrm{~Hz}$ band around $150 \mathrm{~Hz}$. AURIGA is a bar detector equipped with a capacitive resonant transducer, located in Legnaro (PD), Italy. In 2003 the AURIGA detector resumed data acquisition after upgrades that enlarged its sensitive band to $850-950 \mathrm{~Hz}$, from the $\sim 2 \mathrm{~Hz}$ bandwidth of the 1997-1999 run [11-13].

Due to the different spectral shapes, an interferometer-bar coincident search is only sensitive to signals with power in the bar's narrower band. The LIGO-AURIGA analysis thus focused on short duration ( $\leqslant 20 \mathrm{~ms}$ ) transients in the $850-950 \mathrm{~Hz}$ band, with potential target sources like black hole ringdowns [2] and binary black hole mergers [15, 16].

Another important difference between bars and interferometers is the sky coverage, which depends on the detectors' shape and orientation. Figure 1 shows the antenna pattern magnitude $\sqrt{F_{+}^{2}+F_{\times}^{2}}$ of the AURIGA and LIGO Hanford (LHO) detectors, as a function of latitude and longitude. Since directions of maximum LIGO sensitivity overlap with the larger portion of the sky visible to AURIGA, a coincident search is not penalized by differences in antenna pattern. However, adding AURIGA to the detector network does not improve its overall sky coverage either, due to the AURIGA sensitivity, which is $\sim 3$ times worse than LIGO [17, 18].

Despite the different sensitivity and bandwidth, a coincident analysis between LIGO and AURIGA has the potential to suppress false alarms in the LIGO network, thus increasing the confidence in the detection of loud signals and making source localization possible, with triangulation. Collaborative searches also increase the amount of observation time with three or more operating detectors. For this reason, and to bring together the expertise of two traditions in burst analysis, the AURIGA and LIGO Scientific Collaborations pursued a joint search.

The analysis described in this paper follows the all-sky approach described in [18], where data from two or three LIGO interferometers are cross-correlated at the time of AURIGA candidate events. This method was tested on data from the first AURIGA and LIGO coincident run, a 389 h period between 24 December 2003 and 9 January 2004, during the third LIGO science run S3 [7] and the first run of the upgraded AURIGA detector [12, 13]. Only a 

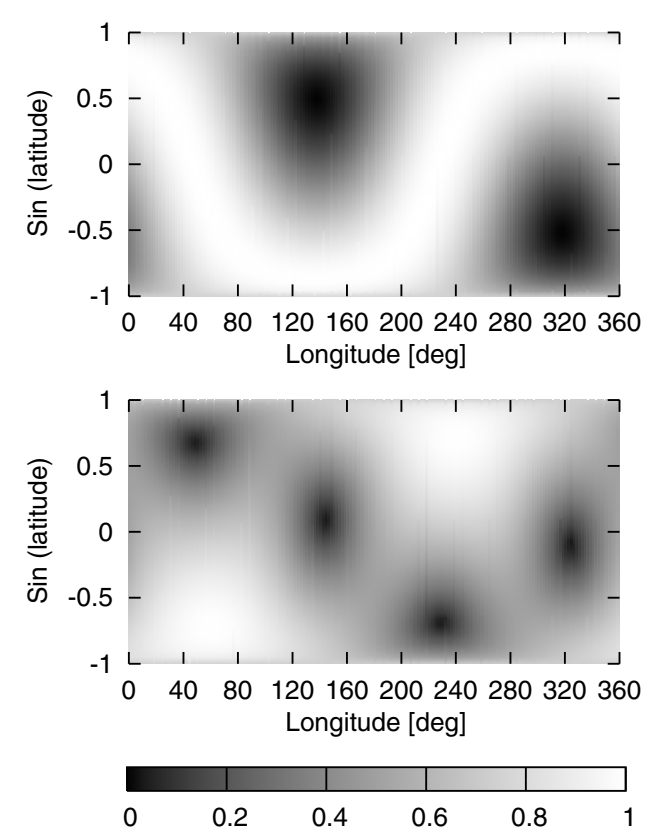

Figure 1. Antenna pattern magnitude as a function of the longitude and the sine of the latitude. Top: AURIGA; bottom: LIGO Hanford.

portion of these data was used in the joint burst search, because of the detectors' duty factors and the selection of validated data segments which was independently performed by the two collaborations [17, 19]. The effective livetime available for the analysis was

- $36 \mathrm{~h}$ of 4-fold coincidence between AURIGA and the three LIGO interferometers;

- $74 \mathrm{~h}$ of 3-fold coincidence between AURIGA and the two LIGO Hanford interferometers, when data from the LIGO Livingston detector were not available.

Other three-detector combinations including AURIGA were not considered, due to the low duty factor of the LIGO Livingston interferometer in S3. The 4-fold and 3-fold data sets were separately analyzed and the outcome was combined into a single result.

Figure 2 shows the best single-sided sensitivity spectra for LIGO and AURIGA in the $800-1000 \mathrm{~Hz}$ band at the time of the coincident run. The AURIGA spectrum contained spurious lines, due to the up-conversion of low frequency seismic noise. These lines were non-stationary and could not always be filtered by the AURIGA data analysis; for this reason, a large portion of the data (up to $42 \%$ ) had to be excluded from the analysis, with significant impact on the livetime [17, 19]. The largest peak in each LIGO spectrum is a calibration line, filtered in the analysis. The amount of available LIGO livetime was limited by several data quality factors, such as data acquisition problems, excessive dust at the optical tables and fluctuations of the light stored in the cavities, as described in [7].

Due to the short duration of the coincidence run and the non-optimal detector performances, the work described in this paper has a methodological relevance. On the other hand, it is worth pointing out that the 3-fold coincidence between AURIGA and the two Hanford interferometers, when Livingston was offline, allowed the exploration of some data that would not have been searched otherwise. 


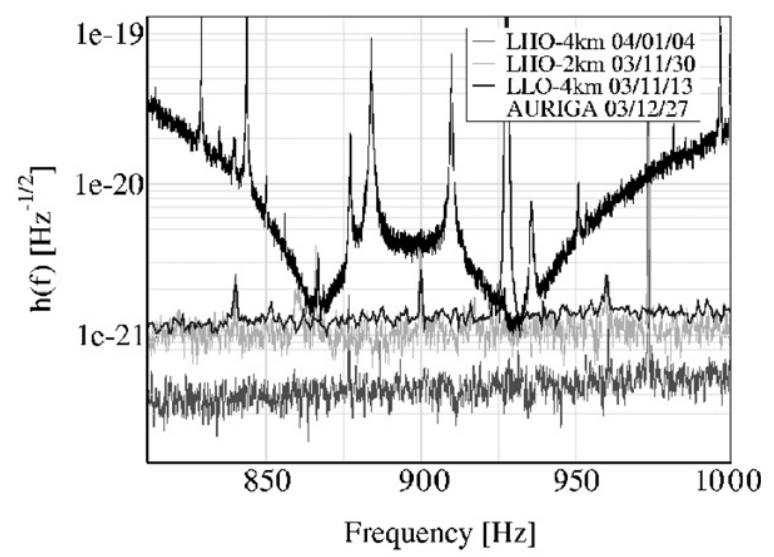

Figure 2. Best single-sided sensitivity spectra of AURIGA and the three LIGO interferometers during the joint observation. In the AURIGA spectrum, several spurious lines are visible while LIGO spectra present calibration lines at $973 \mathrm{~Hz}$ for the Hanford detectors (LHO $4 \mathrm{~km}$ and LHO $2 \mathrm{~km}$ ) and $927 \mathrm{~Hz}$ for the Livingston detector (LLO $4 \mathrm{~km}$ ).

\section{The analysis pipeline}

The joint analysis followed a statistically blind procedure to avoid biases on the result: the pipeline was tested, thresholds were fixed and procedural decisions were made before the actual search, according to the following protocol $[18,19]$.

(i) AURIGA provided a list of burst candidates (triggers) in the validated observation time. The triggers were identified by matched filtering to a $\delta$-like signal, with signal-to-noise ratio threshold SNR $\geqslant 4.5$. Triggers at lower SNR were not included in this analysis, since their rate and time uncertainty increased steeply to unmanageable levels, with negligible improvement in detection efficiency. Special attention was required, in this run, to address non-stationary noise with data quality vetoes that were not needed in subsequent runs $[17,19]$. The resulting events were auto-correlated up to about $300 \mathrm{~s}$; this effect, particularly evident for high SNR events, was due to an imperfect suppression of the non-stationary spurious lines on short time scales.

(ii) Data from the three LIGO interferometers at the time of AURIGA triggers were crosscorrelated by the $r$-statistic waveform consistency test [20], a component of the LIGO burst analysis [6,7] performed with the CorrPower code [21]. The test compares the broadband linear cross-correlation $r$ between two data streams to the normal distribution expected for uncorrelated data and computes its $p$-value, the probability of getting a larger $r$ if no correlation is present, expressed as $\Gamma=-\log _{10}(p$-value $)$. When more than two streams are involved, $\Gamma$ is the arithmetic mean of the values for each pair. The cross-correlation was performed on 20,50 and $100 \mathrm{~ms}$ integration windows, to allow for different signal durations. Since the source direction was unknown, the integration windows were slid around each AURIGA trigger by $27 \mathrm{~ms}+\sigma_{t}$, sum of the light travel time between AURIGA and Hanford and of the estimated $1 \sigma$ timing error of the AURIGA trigger. The value of $\sigma_{t}$ depended on the SNR of each trigger, typically in the 5-40 ms range, with an average value of $17 \mathrm{~ms}$. The resulting $\Gamma$ was the maximum amongst all time slides and integration windows. Only triggers above the minimal analysis threshold of $\Gamma \geqslant 4$ were considered as coincidences. 
(iii) A selection cut was applied on the sign of the correlation between the two LIGO Hanford interferometers, which must be positive for a gravitational wave signal in the two colocated detectors. This cut, also used in the LIGO-only analysis [7], reduced by a factor $\sim 2$ the number of accidental coincidences, with no effect on the detection efficiency.

(iv) The data analysis pipeline was first applied, for testing purposes, to a playground data set [22], which amounted to about $10 \%$ of the livetime and was later excluded from the data set used in the analysis.

(v) To explore the false alarm statistics, we produced an off-source data set by introducing an unphysical time delay in the data from different LIGO sites. With analogous nomenclature, we refer to the unshifted data as the on-source set. Details on how the off-source set was used to estimate the background statistics are provided in section 2.1.

(vi) The detection efficiency was estimated with Monte Carlo methods [19]. Simulated gravitational wave signals from a population of sources, uniformly distributed in the sky and in polarization angle, were added to the output of each detector, and the resulting time series were analyzed through the same analysis pipeline. A description of the waveforms and the procedure is provided in section 2.2.

(vii) The analysis tuning consisted of setting two thresholds: on the SNR of the AURIGA candidate events and on the LIGO $\Gamma$ value. Details on the tuning procedure are available in section 2.3.

(viii) The statistical analysis plan was defined a priori, with decisions on which combination of detectors to analyze (4-fold and 3-fold) and how to merge the results, the confidence level for the null hypothesis test, and the procedure to build the confidence belt, as described in section 2.5 .

(ix) Once analysis procedure and thresholds were fixed, the search for gravitational wave bursts was applied to the on-source data set. The statistical analysis led to confidence intervals which were interpreted as rate upper limit versus amplitude curves. A posteriori investigations were performed on the on-source results (see section 3.1), but these followup studies did not affect the statistical significance of the a priori analysis.

\subsection{Accidental coincidences}

The statistics of accidental coincidences were studied on independent off-source data sets, obtained with unphysical time shifts between data from the Livingston and Hanford LIGO detectors and AURIGA. The two Hanford detectors were not shifted relative to each other, to account for local Hanford correlated noise. The shifts applied to each LIGO site were randomly chosen between $7 \mathrm{~s}$ and $100 \mathrm{~s}$, with a minimum separation of $1 \mathrm{~s}$ between shifts. Hanford-Livingston shifts in the 4-detector search also had to differ by more than $1 \mathrm{~s}$. The livetime in each shifted set varied by a few per cent due to the changing combination of data quality cuts in the various detectors. The net livetime used in the accidental rate estimate was $2476.4 \mathrm{~h}$ from 74 shifts in the 4-detector search and $4752.3 \mathrm{~h}$ from 67 shifts in the three-detector search.

Figure 3 shows scatter plots of the LIGO $\Gamma$ versus the AURIGA SNR for background events surviving the cut on the Hanford-Hanford correlation sign, in the 4-detector and in the 3 -detector configurations. The regions at SNR $<4.5$ and $\Gamma<4$ are shaded, as they are below the minimal analysis threshold.

The number of off-source accidental coincidences in each time shift should be Poisson distributed if the time-slide measurements are independent of each other. For quadruple and for triple coincidences, a $\chi^{2}$-test compared the measured distributions of the number of accidentals to the Poisson model. The test included accidental coincidences with $\Gamma \geqslant 4$ 

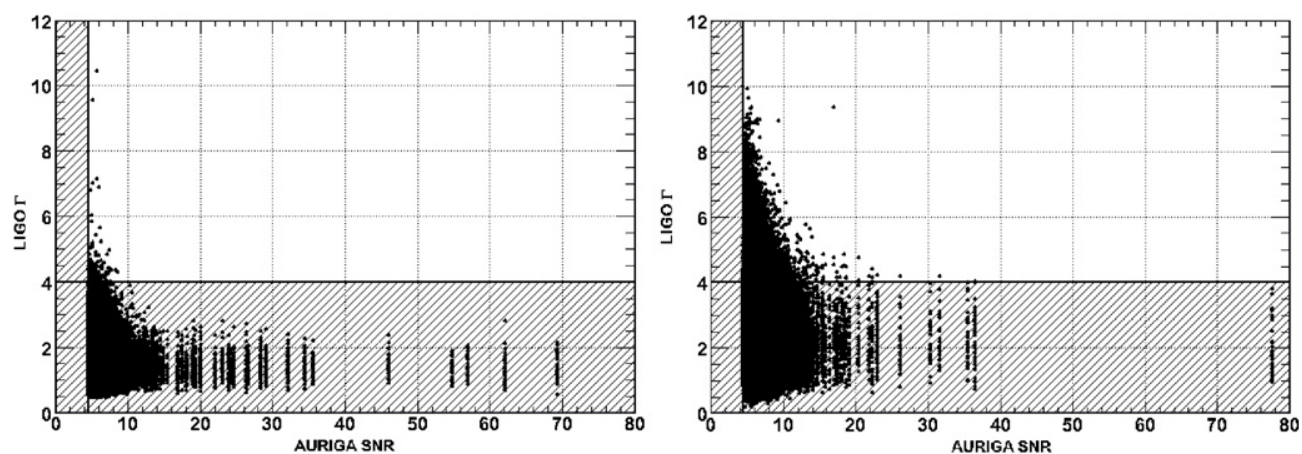

Figure 3. Scatter plots of the LIGO $\Gamma$ versus the AURIGA SNR for background events surviving the cut on the sign of the $\mathrm{H} 1-\mathrm{H} 2$ correlation, in the 4-detector (left) and in the 3-detector (right) configurations. The regions at SNR $<4.5$ and $\Gamma<4$ are shaded, as they are below the minimal analysis threshold and were not used in the tuning. The vertical structures at large SNR and $\Gamma<4$ are an artifact of the off-source data set. For each AURIGA event, there are several instances of cross-correlation of LIGO data at different time shifts. Each time shift thus contributes a point in the scatter plot, with the same SNR and a distribution of $\Gamma$ values; the vertical structures are associated with AURIGA outliers.

and $\Gamma \geqslant 7.5$ for 4 -fold and 3-fold coincidences, respectively. These thresholds were lower than what was used in the coincidence search (section 2.3), to ensure a sufficiently large data sample, while the AURIGA threshold remained at SNR $\geqslant 4.5$. The corresponding $p$-values were $34 \%$ and $6.5 \%$, not inconsistent with the Poisson model for the expected number of accidentals.

\subsection{Network detection efficiency}

The detection efficiency was estimated by adding software-generated signals to real data, according to the LIGO Mock Data Challenge procedure [23]. The simulation generated gravitational waves from sources isotropically distributed in the sky, with azimuthal coordinate uniform in $[0,2 \pi]$, cosine of the polar sky coordinate uniform in $[-1,1]$ and wave polarization angle uniform in $[0, \pi]$. Three waveform classes were considered $[17,19]$ :

(i) Gaussians with linear polarization:

$$
\left\{\begin{array}{l}
h_{+}(t)=h_{\text {peak }} \mathrm{e}^{-\left(t-t_{0}\right)^{2} / \tau^{2}} \\
h_{\times}(t)=0
\end{array}\right.
$$

with $\tau=0.2 \mathrm{~ms}$.

(ii) sine-Gaussians with linear polarization:

$$
\left\{\begin{array}{l}
h_{+}(t)=h_{\text {peak }} \mathrm{e}^{-\left(t-t_{0}\right)^{2} / \tau^{2}} \sin \left(2 \pi f_{0}\left(t-t_{0}\right)\right) \\
h_{\times}(t)=0
\end{array}\right.
$$

with $f_{0}=900 \mathrm{~Hz}, \tau=2 / f_{0}=2.2 \mathrm{~ms}$ and $Q \equiv \sqrt{2} \pi f_{0} \tau=8.9$. In this analysis we also tested cosine-Gaussians (with the sine replaced by cosine), and found the same sensitivity as for sine-Gaussian waveforms. 

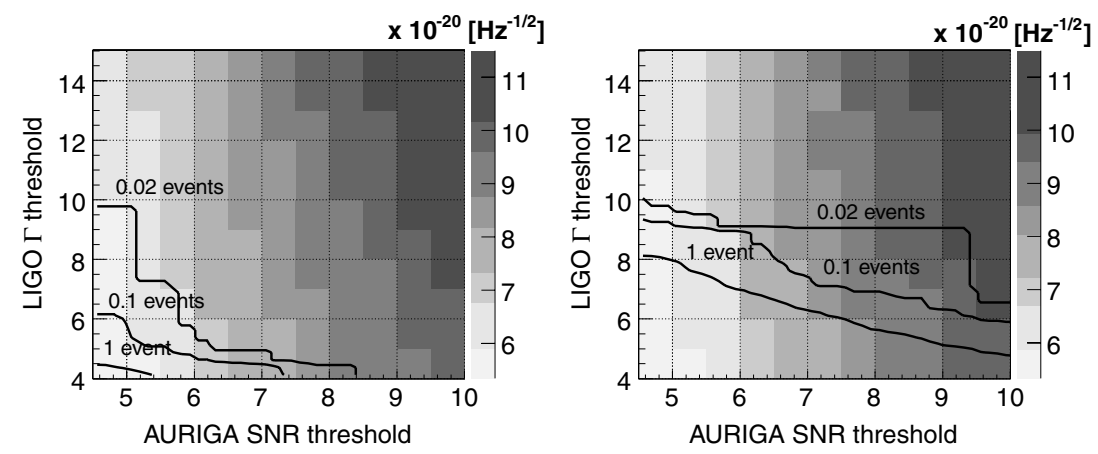

Figure 4. Grayscale plot: efficiency to sine-Gaussians waveforms in terms of $h_{\mathrm{rss}} 50 \%$. Left: 4 fold; right: 3 -fold observations. The contour lines indicate the number of accidental coincidences expected in the on-source data set.

(iii) Damped sinusoids with circular polarization:

$$
\begin{aligned}
& h_{+}(t)= \begin{cases}h_{\text {peak }} \frac{1+\cos ^{2} \iota}{2} \cos \left[2 \pi f_{0}\left(t-t_{0}\right)\right] \mathrm{e}^{-\left(t-t_{0}\right) / \tau} & t-t_{0} \geqslant 0, \\
h_{\text {peak }} \frac{1+\cos ^{2} \iota}{2} \cos \left[2 \pi f_{0}\left(t-t_{0}\right)\right] \mathrm{e}^{10\left(t-t_{0}\right) / \tau} & t-t_{0}<0,\end{cases} \\
& h_{\times}(t)= \begin{cases}h_{\text {peak }} \cos \iota \sin \left[2 \pi f_{0}\left(t-t_{0}\right)\right] \mathrm{e}^{-\left(t-t_{0}\right) / \tau} & t-t_{0} \geqslant 0 \\
h_{\text {peak }} \cos \iota \sin \left[2 \pi f_{0}\left(t-t_{0}\right)\right] \mathrm{e}^{10\left(t-t_{0}\right) / \tau} & t-t_{0}<0\end{cases}
\end{aligned}
$$

with $f_{0}=930 \mathrm{~Hz}, \tau=6 \mathrm{~ms}$ and $\cos \iota$ uniformly distributed in $[-1,1], \iota$ being the inclination of the source with respect to the line of sight. $\mathrm{e}^{10\left(t-t_{0}\right) / \tau}$ is an empirical smoothing factor to connect the damped sinusoid with the noise floor.

Although no known astrophysical source is associated with Gaussian and sine-Gaussian waveforms, they are useful because of their simple spectral interpretation and they are standard test waveforms in LIGO burst searches. Damped sinusoids are closer to physical templates $[2,15,16]$.

The signal generation was performed with the LIGO LDAS software [24]; the waveforms were added to calibrated LIGO and AURIGA data and the result was analyzed by the same pipeline used in the search. For each waveform class, the simulation was repeated at different signal amplitudes to measure the efficiency of the network as a function of the square root of the burst energy,

$$
h_{\mathrm{rss}}=\sqrt{\int_{-\infty}^{\infty} \mathrm{d} t\left[h_{+}^{2}(t)+h_{\times}^{2}(t)\right]}=\sqrt{2 \int_{0}^{\infty} \mathrm{d} f\left[\tilde{h}_{+}^{2}(f)+\tilde{h}_{\times}^{2}(f)\right]} .
$$

\subsection{Analysis tuning}

The analysis thresholds were chosen to maximize the detection efficiency with an expected number of accidental coincidences smaller than 0.1 in each of the 3- and 4-detector searches. Figure 4 shows contour plots of the number of accidental coincidences expected in the onsource data set, the original un-shifted data that may include a gravitational wave signal, as a function of the $\Gamma$ and SNR thresholds. This quantity is the number of accidental coincidences found in the time shifted data, scaled by the ratio of on-source to off-source livetimes. The 
Table 1. Signal amplitudes with $50 \%$ and $90 \%$ detection efficiency in the 4-fold and 3-fold searches at the chosen analysis thresholds of SNR $\geqslant 4.5$ and $\Gamma \geqslant 6$ (4-fold) or $\Gamma \geqslant 9$ (3-fold). These numbers do not include a $\sim 10 \%$ systematic error, due to calibration uncertainties, and a $\sim 3 \% 1 \sigma$ statistical error, due to the small number of simulated signals.

\begin{tabular}{|c|c|c|c|c|}
\hline \multirow[b]{2}{*}{ Waveform } & \multicolumn{2}{|c|}{$h_{\mathrm{rss}} 50 \%\left(10^{-20} \mathrm{~Hz}^{-1 / 2}\right)$} & \multicolumn{2}{|c|}{$h_{\mathrm{rss}} 90 \%\left(10^{-19} \mathrm{~Hz}^{-1 / 2}\right)$} \\
\hline & 4-fold & 3 -fold & 4-fold & 3 -fold \\
\hline Sine-Gaussians & 5.6 & 5.8 & 4.9 & 5.3 \\
\hline Gaussians & 15 & 15 & 10 & 11 \\
\hline Damped sinusoids & 5.7 & 5.7 & 3.3 & 3.4 \\
\hline
\end{tabular}

plots also show the detectability of sine-Gaussian waveforms, expressed as $h_{\mathrm{rss}} 50 \%$, the signal amplitude with $50 \%$ detection probability.

For all tested waveforms, the detection efficiencies in the 4-fold and 3-fold searches are the same, within $10 \%$; their value is dominated by the AURIGA sensitivity at SNR $\geqslant 4.5$. This observation, together with the shape of the accidental rate contour plots, indicates that the best strategy for the suppression of accidental coincidences with minimal impact on detection efficiency is to increase the $\Gamma$ threshold and leave the SNR threshold at the exchange value of 4.5. The analysis thresholds were chosen to yield the same accidental rate in the two data sets: $\Gamma \geqslant 6$ and SNR $\geqslant 4.5$ for the 4 -fold search and $\Gamma \geqslant 9$ and SNR $\geqslant 4.5$ for the 3 -fold search.

It was decided a priori to quote a single result for the entire observation time by merging the 4 -fold and the 3 -fold periods. The number of expected accidental coincidences in the combined on-source data set is 0.24 events in $110.0 \mathrm{~h}$, with a $1 \sigma$ statistical uncertainty of 0.06 . Detection efficiencies with the chosen thresholds are listed in table 1.

\subsection{Error propagation}

The detection efficiency, in a coincidence analysis, is dominated by the least sensitive detector, in this case AURIGA. Monte Carlo efficiency studies show that only a small fraction of the simulated events are lost due to the LIGO $\Gamma$ threshold; these events were missed because their sky location and polarization were in an unfavorable part of LIGO's antenna pattern. Since most of the simulated events were cut by the AURIGA threshold, the main source of systematics in this analysis is the calibration error in AURIGA data, estimated to be $\sim 10 \%$.

In addition, there is a statistical error due to the simulation statistics and to the uncertainty in the asymptotic number of injections after the veto implementation. The $1 \sigma$ statistical error in the numbers in table 1 is about $3 \%$.

Both systematic and statistical errors were taken into account in the final exclusion curve in figure 7. The statistical error is propagated from the fit of the efficiency curve to a 4parameter sigmoid [6-8]. The fit parameters were worsened to ensure a $90 \%$ confidence level in the fit, following the prescriptions in [27]. An additional, conservative shift to the left was applied to account for the $10 \%$ error in the calibration uncertainty, which is the dominant error.

\subsection{Statistical interpretation plan}

In compliance with the blind analysis approach, the statistical interpretation was established a priori. The procedure is based on a null hypothesis test to verify that the number of on-source coincidences is consistent with the expected distribution of accidentals, a Poisson with mean 


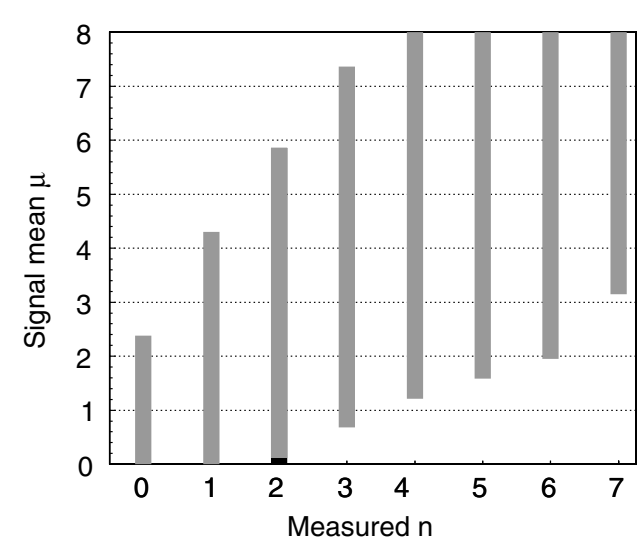

Figure 5. Confidence belt based on the Feldman and Cousins procedure, with $90 \%$ C.L., unknown Poisson signal mean $\mu$ and a Poisson background with $b=0.24 \pm 3 \sigma$. The standard Feldman and Cousins construction (solid lines) is modified by fixing the maximum false alarm probability to $1 \%$ : the lower bound is fixed to 0 for 2 measured events.

0.24 . We require a $99 \%$ test significance, which implies the null hypothesis is rejected if at least three coincidences are found.

The set of alternative hypotheses is modeled by a Poisson distribution:

$$
P(n \mid \mu)=(\mu+b)^{n} \exp [-(\mu+b)] / n !,
$$

where the unknown $\mu$ is the mean number of counts in excess of the accidental coincidences, which could be due to gravitational waves, to environmental couplings or to instrumental artifacts. Confidence intervals are established by the Feldman and Cousins method with 90\% coverage [25]. Uncertainties in the estimated accidental coincidence number are accounted for by taking the union of the two confidence belts with $b=0.24 \pm 3 \sigma$, with $\sigma=0.06$.

The confidence belt was modified to control the false alarm probability according to the prescription of the null hypothesis test: if less than three events are found, and the null hypothesis is confirmed at $99 \%$ C.L., we accept the upper bound of the Feldman and Cousins construction but we extend its lower bound to 0 regardless of the belt value. The resulting confidence belt, shown in figure 5, is slightly more conservative than the standard Feldman and Cousins belt for small values of the signal $\mu$. The advantage of this modification is to separate the questions of what is an acceptable false detection probability and what is the required minimum coverage of the confidence intervals [26].

An excess of on-source coincidences could be due to various sources, including instrumental and environmental correlations; the rejection of the null hypothesis or a confidence interval on $\mu$ detaching from zero do not automatically imply a gravitational wave detection. A detection claim requires careful follow-up studies, to rule out all known sources of foreground, or independent evidence to support the astrophysical origin of the signal. On the other hand, an upper limit on $\mu$ can be interpreted as an upper limit on the number of GWs; therefore, the upper bound of the confidence interval can be used to construct exclusion curves.

\section{Results}

The final step consists of analyzing the on-source data sets. No gravitational wave candidates were found in this search, consistent with the null hypothesis. The resulting $90 \%$ C.L. upper 


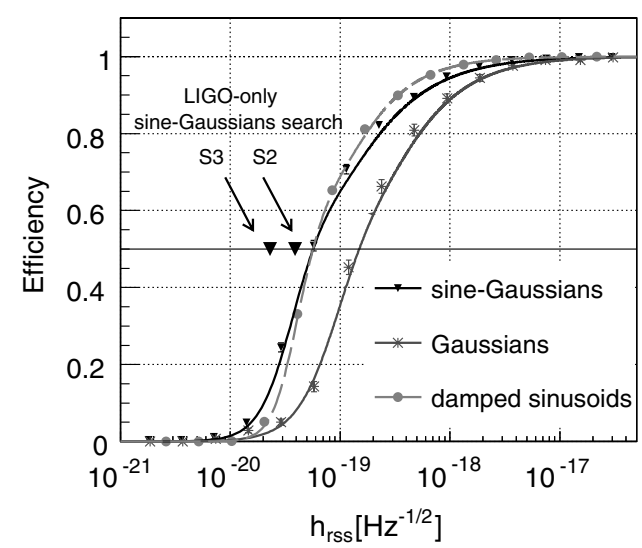

Figure 6. Average efficiency of detection (4-fold +3 -fold) versus $h_{\mathrm{rss}}$ for the considered waveforms: sine-Gaussians at $f_{0}=900 \mathrm{~Hz}$ and $\tau=2.2 \mathrm{~ms}$, Gaussians with $\tau=0.2 \mathrm{~ms}$, and damped sinusoids with $f_{0}=930 \mathrm{~Hz}$ and damping time $\tau=6 \mathrm{~ms}$. All sources have been modeled as uniformly distributed in the sky and with random polarizations (see section 2.2). The triangles mark $h_{\mathrm{rss}} 50 \%$ for sine-Gaussians achieved in the LIGO-only S2 and S3 searches.

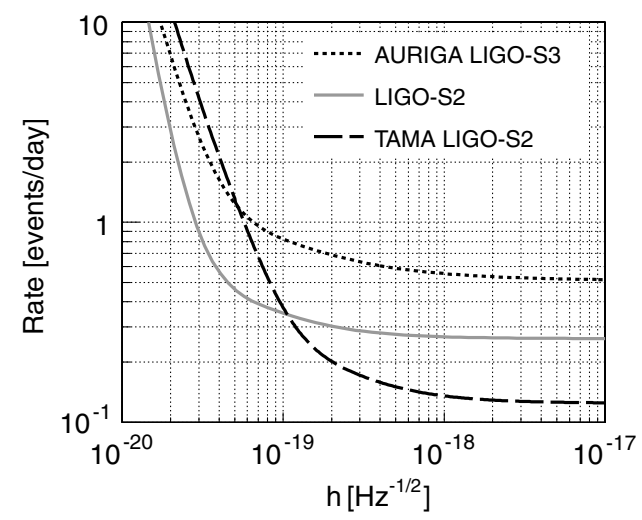

Figure 7. Upper limits at $90 \%$ C.L. on the gravitational wave rate versus $h_{\text {rss }}$ for sine-Gaussian waveforms in different network analyses: AURIGA-LIGO S3 (dotted line), LIGO-only S2 (solid line) and TAMA-LIGO S2 (dashed line). The sine-Gaussians have central frequency $900 \mathrm{~Hz}$ (for AURIGA-LIGO S3) and $850 \mathrm{~Hz}$ (for LIGO-only S2 and TAMA-LIGO S2). In all cases, $Q=8.9$. All sources have been modeled as uniformly distributed in the sky and with random polarizations (see section 2.2).

limit is 2.4 events in the on-source data set, or 0.52 events/day in the combined 3-fold and 4-fold data sets.

Figure 6 shows the combined efficiency for this search as a function of the signal amplitude for the waveforms described in section 2.2, a weighted average of the detection efficiency of 3-fold and 4-fold searches:

$$
\varepsilon\left(h_{\mathrm{rss}}\right)_{4 \text { fold }+3 \text { fold }}=\frac{\varepsilon\left(h_{\mathrm{rss}}\right)_{4 \text { fold }} \cdot T_{4 \text { fold }}+\varepsilon\left(h_{\mathrm{rss}}\right)_{3 \text { fold }} \cdot T_{3 \text { fold }}}{T_{4 \text { fold }}+T_{3 \text { fold }}} .
$$

The $90 \%$ C.L. rate upper limit, divided by the amplitude-dependent efficiency, yields upper limit exclusion curves similar to those obtained in previous searches $[6,10]$. Figure 7 compares the sine-Gaussian exclusion curves found in this search to those from $\mathrm{S} 2$ in LIGO 

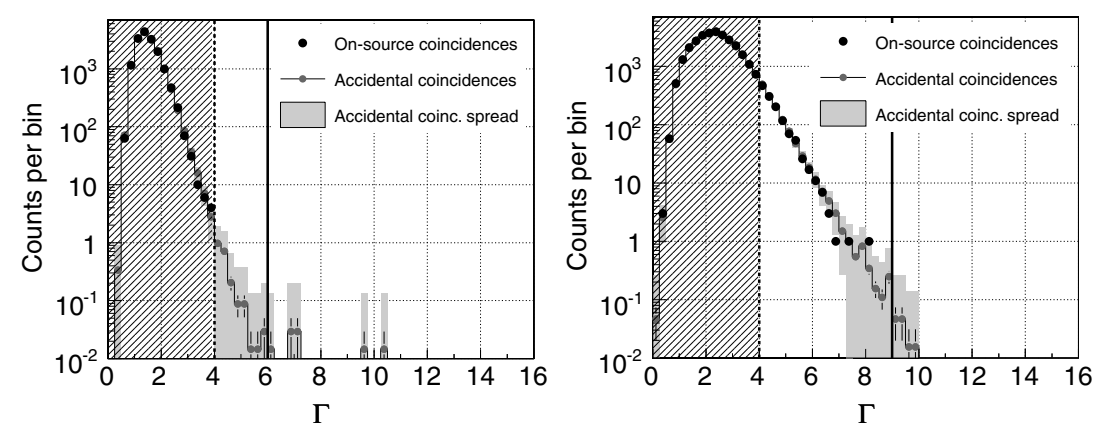

Figure 8. Comparison of $\Gamma$ distributions for 4-fold (left) and 3-fold coincidences (right). The black dots correspond to on-source coincidences. The stair-step curve with gray dots is the mean accidental background, estimated from off-source coincidences, with its $1 \sigma$ RMS spread (shaded gray area) and the error in the mean (thin error bars). The on-source and off-source distributions are in good agreement, within the statistical uncertainty. The solid vertical lines correspond to the analysis thresholds ( $\Gamma=6$ and $\Gamma=9$, respectively). The analysis was tuned only on events with $\Gamma \geqslant 4$ (dotted vertical lines).

and LIGO-TAMA. The waveform used here peaks at $900 \mathrm{~Hz}$, while the previous searches used a sine-Gaussian with $850 \mathrm{~Hz}$ central frequency. We verified analytically that the AURIGA detection efficiencies for $Q=9$ sine-Gaussians at $850 \mathrm{~Hz}$ and $900 \mathrm{~Hz}$ agree within $10 \%$; no significant difference is to be expected for the large band detectors.

The asymptotic upper limit for large amplitude signals is inversely proportional to the observation time. The value for this search with $90 \%$ C.L. is 0.52 events/day, to be compared to 0.26 events/day in the LIGO S2 search [6] and 0.12 events/day in the LIGO-TAMA search [10]. The lowest asymptotic value was previously set by IGEC: $\sim 4 \times 10^{-3}$ events/day, thanks to their longer observation time [4].

The detection efficiency in this search is comparable to the LIGO-only S2 one, and a factor 2 worse than the LIGO-only S3 sensitivity. In the lower amplitude region, this search is an improvement over the IGEC search, since the AURIGA amplitude sensitivity during LIGO S3 was about three times better than the typical bar sensitivity in the IGEC 1997-2000 campaign (a direct comparison is not possible since IGEC results are not interpreted in terms of a source population model). More recent data yielded significant improvements in sensitivities, by a factor $\sim 10$ for the LIGO S4 run [8] and a factor $\sim 3$ for IGEC-2 [28].

\subsection{Diagnostics of on-source and off-source data sets}

The agreement between on-source and off-source coincidences was tested comparing the $\Gamma$ distributions in figure 8 above the minimal exchange threshold $\Gamma \geqslant 4$ and below the network analysis threshold ( $\Gamma \geqslant 6$ for 4 -fold and $\Gamma \geqslant 9$ for 3 -fold). This a posteriori test did not find a disagreement between on-source and off-source distributions. There were no 4-fold, on-source events with $\Gamma \geqslant 4$. For 3-fold events, the agreement between zero-lag and accidental distributions can be confirmed with a Kolmogorov-Smirnov test that uses the empirical distribution of accidentals as a model, giving a $0.6 p$-value.

In addition, we addressed the question of whether on-source events (foreground) modified the distribution of accidentals (background) and biased our estimate. This is an issue in the 3-fold AU-H1-H2 analysis where only $\mathrm{H} 1$ and $\mathrm{H} 2$ are cross-correlated and the measured background distribution includes instances of Hanford foreground events in accidental 
coincidence with an AURIGA shifted event. As a result, the time-shift method overestimates the number of accidentals. In this search, however, this systematic effect turned out to be negligible: the removal of all background events in accidental coincidence with on-source 3 -fold events with $\Gamma \geqslant 5.5$ and SNR $\geqslant 4.5$, did not significantly affect the $\Gamma$ histogram above threshold.

The same question could be posed in a different way: how would the background histogram change if we had an actual gravitational wave event, with large $\Gamma$ ? On average, the same H1-H2 event occurs accidentally in $\sim 9$ different lags. A loud gravitational wave event, with high correlation between $\mathrm{H} 1$ and $\mathrm{H} 2$ and $\Gamma$ above the noise, say $\Gamma=12$, would have appeared in the background histogram as an excess of $\Gamma \leqslant 12$ events at the same Hanford time. Such an event would not have been missed, but it would have been noted in the tuning stages. The most significant consequence is that the 3 -fold search is not truly blind, since a loud signal would easily manifest itself in the tuning data set.

\section{Conclusions}

This paper describes the first joint search for gravitational wave bursts with a hybrid network composed of a narrow band resonant bar detector and broadband interferometers. This was a rare and valuable opportunity to bring together the expertise of the AURIGA and LSC collaborations and explore common methods on real data. The addition of the AURIGA detector to the LIGO observatory allowed to extend the time coverage of the observations by including also the time periods when only two of the three LIGO detectors were operating simultaneously with AURIGA (AU-H1-H2). This was possible thanks to the false alarm rate suppression contributed by AURIGA. The detection efficiency of this hybrid network for the tested source models was about a factor 2 worse than the LIGO-only efficiency, limited by the AURIGA detector. This cost however, turned out smaller than the amplitude sensitivity ratio between AURIGA and LIGO during S3 for the same signal types (roughly, a factor 3).

Due to the short observation time, the relevance of this study is methodological. The results have been interpreted in terms of source population models and the final upper limits are comparable to those set by LIGO alone in previous observations. This joint analysis followed a statistically blind procedure to allow an unbiased interpretation of the confidence of the results. In particular, the data analysis plan has been fixed a priori and the results are confidence intervals which ensure a minimum coverage together with a more stringent requirement on the maximum false detection probability.

Any future joint search for bursts by interferometric and resonant detectors, on simultaneous long-term observations, would be informed by the techniques developed in the ground-breaking work presented in this paper.

\section{Acknowledgments}

The LIGO Scientific Collaboration (LSC) gratefully acknowledge the support of the United States National Science Foundation for the construction and operation of the LIGO Laboratory and the Science and Technology Facilities Council of the United Kingdom, the Max-PlanckSociety, and the State of Niedersachsen/Germany for support of the construction and operation of the GEO600 detector. The authors also gratefully acknowledge the support of the research by these agencies and by the Australian Research Council, the Council of Scientific and Industrial Research of India, the Istituto Nazionale di Fisica Nucleare of Italy, the Spanish Ministerio de Educación y Ciencia, the Conselleria d'Economia, Hisenda i Innovació of the Govern de les 
Illes Balears, the Scottish Funding Council, the Scottish Universities Physics Alliance, The National Aeronautics and Space Administration, the Carnegie Trust, the Leverhulme Trust, the David and Lucile Packard Foundation, the Research Corporation, and the Alfred P Sloan Foundation. The AURIGA Collaboration acknowledges the support of the research by the Istituto Nazionale di Fisica Nucleare (INFN), the Universities of Ferrara, Firenze, Padova and Trento, the Center of Trento of the Istituto di Fotonica e Nanotecnologie - Istituto Trentino di Cultura and the Consorzio Criospazio Ricerche of Trento. This paper was assigned LIGO document number P070086-04.

\section{References}

[1] Ott C D, Burrows A, Dessart L and Livne E 2006 Phys. Rev. Lett. 96201102

[2] Flanagan É E and Hughes S A 1998 Phys. Rev. D 574535

[3] Flanagan É E and Hughes S A 1998 Phys. Rev. D 574566

[4] Astone P et al 2003 Phys. Rev. D 68022001

[5] Abbott B et al (LIGO Scientific Collaboration) 2004 Phys. Rev. D 69102001

[6] Abbott B et al (LIGO Scientific Collaboration) 2005 Phys. Rev. D 72062001

[7] Abbott B et al (LIGO Scientific Collaboration) 2006 Class. Quantum Grav. 23 S29-39

[8] Abbott B et al (LIGO Scientific Collaboration) 2007 Class. Quantum Grav. 24 5343-69

[9] Ando M et al 2005 Phys. Rev. D 69082002

[10] Abbott B et al 2005 Phys. Rev. D 72122004

[11] Zendri J P et al 2003 Status report of the gravitational wave detector AURIGA Gravitational Waves and Experimental Gravitation, Proc. XXXVIIIth Moriond Workshop ed J Dumarchez and J Tran Thanh Van (Vietnam: The Gioi Publishers) pp 37-42

[12] Vinante A (for the AURIGA Collaboration) 2006 Class. Quantum Grav. 23 S103-10

[13] Baggio L et al 2005 Phys. Rev. Lett. 94241101

[14] Abbott B et al 2007 Phys. Rev. D 76022001

[15] Baker J et al 2002 Phys. Rev. D 65124012

[16] Campanelli M, Lousto C O and Zlochower Y 2006 Phys. Rev. D 73061501

[17] Poggi S 2006 A joint search for gravitational wave bursts by the AURIGA resonant detector and the LIGO interferometer observatories $P h D$ Thesis Università degli Studi di Trento, Italy. Online at: http://www.auriga.Inl.infn.it/auriga/papers_src/phd_poggi.zip

[18] Cadonati L et al 2005 Class. Quantum Grav. 22 S1337-47

[19] Poggi S, Salemi F (for the AURIGA collaboration) and Cadonati L (for the LIGO Scientific collaboration) 2006 J. Phys.: Conf. Ser. 32 198-205

[20] Cadonati L 2004 Class. Quantum Grav. 21 S1695-703

[21] Cadonati L and Márka S 2005 Class. Quantum Grav. 22 S1159-67

[22] Finn L S 2003 S3 Playground Selection, LIGO-T030256-00-Z LIGO Internal Report

[23] Yakushin I, Klimenko S and Rakhmanov M 2004 MDC frames for S2 burst analysis LIGOT040042-00-Z-00 D LIGO Internal Report available in the LIGO Document Control Center at www.ligo.caltech.edu/docs/T/T040042-00.pdf

[24] The LIGO Data Analysis System home page is http://www.ldas-sw.ligo.caltech.edu

[25] Feldman G J and Cousins R D 1998 Phys. Rev. D 57 3873-89

[26] Baggio L and Prodi G A Statistical problems associated with the analysis of data from a network of narrow-band detectors Statistics for Gravitational Wave Data Analysis Center for Gravitational Wave Physics, Penn State University, 19-21 May 2005, http://cgwp.gravity.psu.edu/events/GravStat/Prodi_GravStat.ppt

[27] James F 1994 'MINUIT: Function Minimization and Error Analysis' Reference Manual, Version 94.1 CERN document D506

[28] Astone P et al 2007 Phys. Rev. D 76102001 